\title{
Silica-supported tungsten neosilyl oxo precatalysts: impact of the podality on activity and stability in olefin metathesis.
}

D. Grekov, ${ }^{\ddagger}$ Y. Bouhoute, ${ }^{+}$K. C. Szeto, ${ }^{+}$N. Merle, ${ }^{\dagger}$ A. De Mallmann, ${ }^{\dagger}$ F. Lefebvre, ${ }^{+}$C. Lucas + I. Del Rosal, ${ }^{\perp}$ L. Maron,,${ }^{,}{ }^{*}$ R. M. Gauvin,, L. Delevoye,,${ }^{*}$ M. Taoufik ${ }^{*}$

'Laboratoire de Chimie, Catalyse, Polymères et Procédés, UMR 5265 CNRS/ESCPE-Lyon/UCBL, ESCPE Lyon, F-308-43, Boulevard du 11 Novembre 1918, F-69616 Villeurbanne Cedex, France. Email:mostafa.taoufik@univ-lyon1.fr

tUnité de Catalyse et de Chimie du Solide, CNRS UMR 8181, Université de Lille Nord, F-59655 Villeneuve d'Ascq, France. E-mail : laurent.delevoye@ensc-lille.fr

${ }^{\perp}$ Laboratoire de Physico-Chimie des Nano-Objets, CNRS UMR 5215, Université de Toulouse, INSA, UPS, 135 avenue de Rangueil, F-31077 Toulouse, France. E-mail : laurent.maron@irsamc.ups-tlse.fr

\section{Supporting Information}

\section{Table of contents}

Figure S1. Model of silica surface dehydroxylated at $700^{\circ} \mathrm{C}$.

Figure S2. Optimized structures of mono-grafted complexes

Table S1. Selected DFT-calculated bond lengths of molecular and grafted complexes

Figure S3. Product distribution of propylene self-metathesis over $\mathbf{2 a}$.

Figure S4. Cumulated TON of propylene self-metathesis over $\mathbf{2 a}, \mathbf{2 b}$ and $\mathbf{3 .}$ 


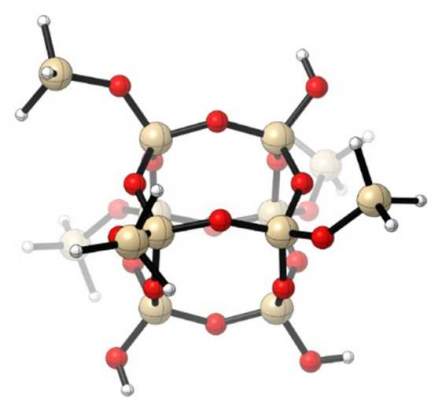

Figure S1. Representation of the used model as silica surface dehydroxylated at $700^{\circ} \mathrm{C}$.

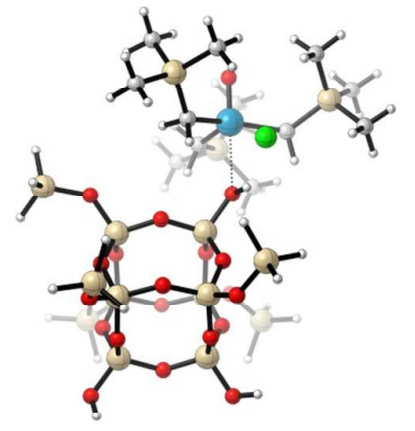

(a) $\mathrm{A}_{\mathrm{HCl}}$

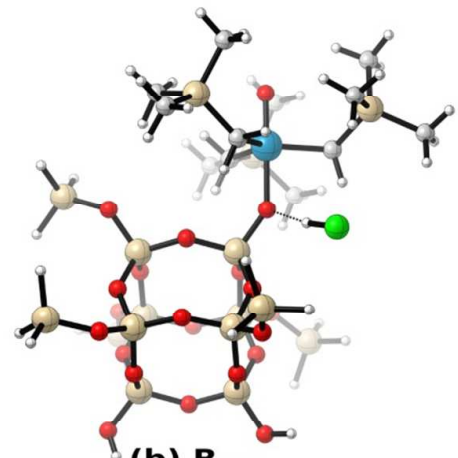

(b) $\mathrm{B}_{\mathrm{HCl}}$

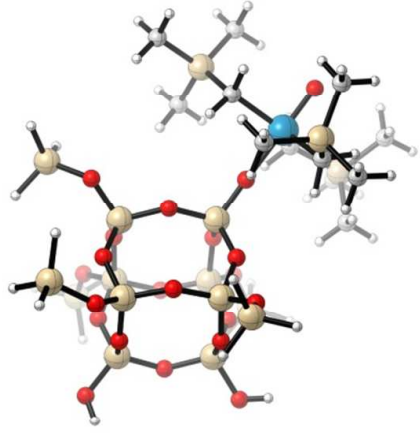

(c) 2a-calc

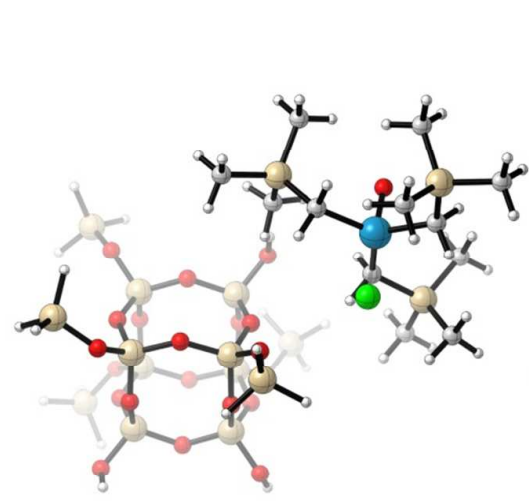

(d) $A_{\text {TMS }}$

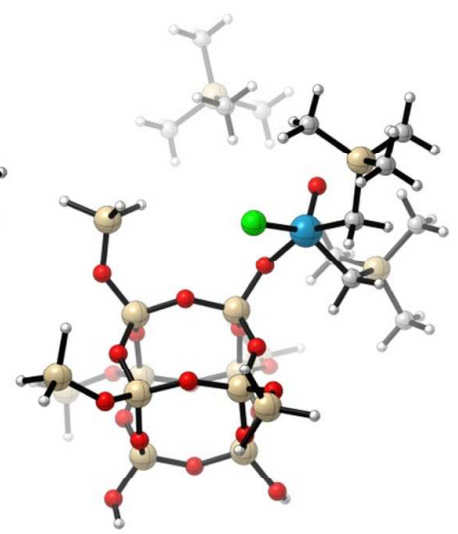

(e) $B_{\text {TMS }}$

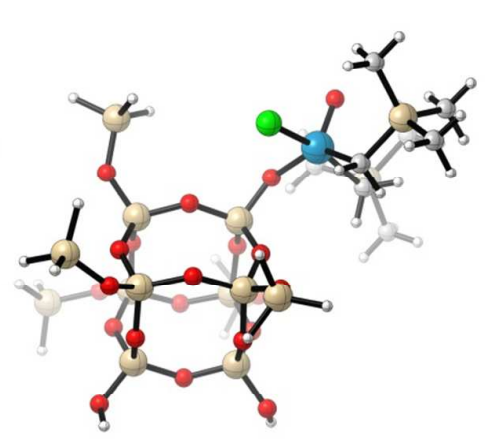

(f) 4-calc

Figure S2. Optimized structures of mono-grafted complexes involved in the grafting reaction of 1 onto c model. 
Table S1. Selected DFT-calculated bond lengths $(\AA)$ of molecular and grafted complexes on the $\mathrm{SiO}_{2-200}$ c model.

\begin{tabular}{|c|c|c|c|c|c|c|c|c|}
\hline & $\mathrm{W}=\mathrm{O}$ & $\underline{\mathrm{W}}-\underline{\mathrm{OS}} \equiv$ & $\underline{\mathrm{W}}-\mathrm{OS} \underline{\mathrm{S}} \equiv$ & $\mathrm{W}-\mathrm{Cl}$ & $\underline{\mathrm{W}}-\underline{\mathrm{C}} \mathrm{H}_{2} \mathrm{SiMe}_{3}$ & $\underline{\mathrm{H}}-\underline{\mathrm{OS}} \mathrm{S} \equiv$ & $\underline{\mathrm{Cl}-\mathrm{H}}-\mathrm{OSi} \equiv$ & $\equiv \mathrm{Si}-\mathrm{O}-\underline{\mathrm{H}}-\mathrm{CH}_{2} \mathrm{SiMe}_{3}$ \\
\hline \multicolumn{9}{|c|}{ Molecular Complex } \\
\hline 1 & 1.703 & - & - & 2.456 & $2.100^{(1)}$ & - & - & - \\
\hline \multicolumn{9}{|c|}{ Mono-grafted complexes } \\
\hline $\mathrm{A}_{\mathrm{HCl}}$ & 1.684 & 2.600 & 4.041 & 2.653 & $\begin{array}{l}2.098 \\
2.111 \\
2.119\end{array}$ & 0.989 & 2.072 & - \\
\hline $\operatorname{TS}\left(\mathbf{A}_{\mathrm{HCl}} \rightarrow \mathbf{B}_{\mathrm{HCl}}\right)$ & 1.687 & 2.361 & 3.833 & 3.195 & $\begin{array}{l}2.093 \\
2.086 \\
2.104\end{array}$ & 1.046 & 1.786 & - \\
\hline $\mathbf{B}_{\mathrm{HCl}}$ & 1.710 & 2.061 & 3.489 & 4.289 & $\begin{array}{l}2.097 \\
2.100 \\
2.102\end{array}$ & 1.899 & 1.309 & - \\
\hline 2a-calc & 1.712 & 1.995 & 3.612 & - & $\begin{array}{l}2.103 \\
2.103 \\
2.104\end{array}$ & - & - & - \\
\hline $\mathbf{A}_{\mathrm{TMS}}$ & 1.705 & 6.020 & 6.715 & 2.458 & $\begin{array}{l}2.088 \\
2.105 \\
2.108\end{array}$ & 0.965 & - & - \\
\hline $\mathrm{TS}\left(\mathbf{A}_{\mathrm{TMS}} \rightarrow \mathbf{B}_{\mathrm{TMS}}\right)$ & 1.704 & 2.326 & 3.718 & 2.437 & $\begin{array}{l}2.077 \\
2.090 \\
2.352\end{array}$ & 1.284 & - & 1.304 \\
\hline $\mathbf{B}_{\mathrm{TMS}}$ & 1.693 & 1.910 & 3.429 & 2.343 & $\begin{array}{l}2.133 \\
2.144 \\
7.543\end{array}$ & - & - & 1.095 \\
\hline 4-calc & 1.692 & 1.891 & 3.347 & 2.372 & $\begin{array}{l}2.115 \\
2.165\end{array}$ & - & - & - \\
\hline
\end{tabular}




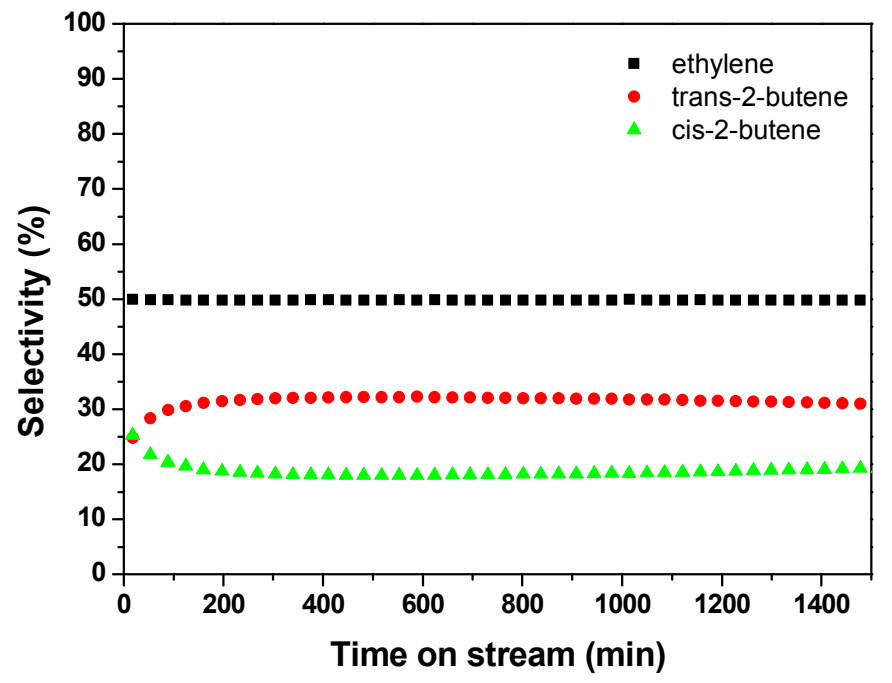

Figure S3. Product distribution of propylene self-metathesis $\left(20 \mathrm{~mL}_{\mathrm{C} 3 \mathrm{H} 6} \min ^{-1} ; 80{ }^{\circ} \mathrm{C} ; 60 \mathrm{~mol}_{\mathrm{C} 3 \mathrm{H} 6}\right.$ $\mathrm{mol}_{\mathrm{W}}{ }^{-1} \mathrm{~min}^{-1}, 1$ bar) over $\mathbf{2 a}$.

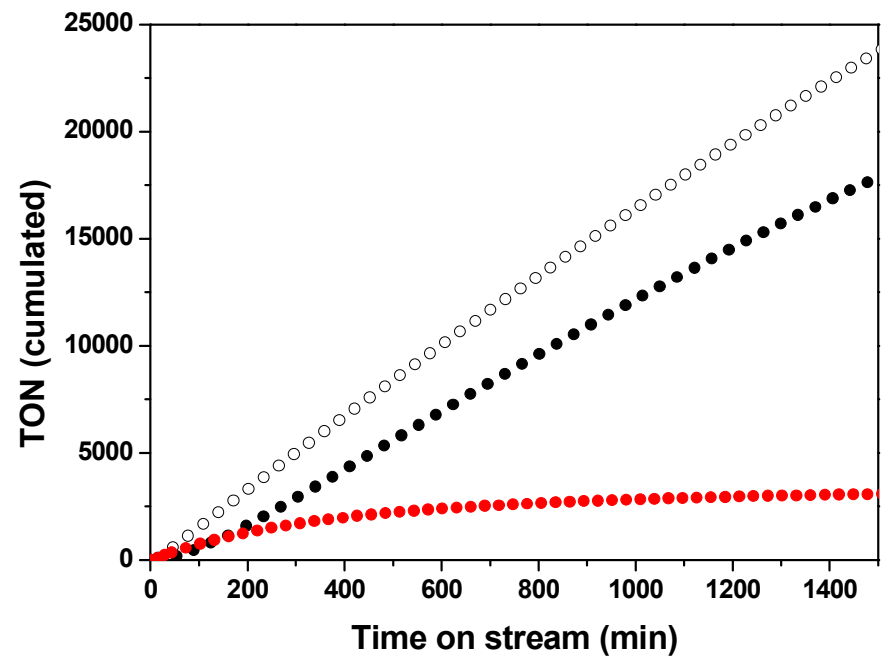

Figure S4. Cumulated TON of propylene self-metathesis $\left(20 \mathrm{~mL}_{\mathrm{C} 3 \mathrm{H} 6} \mathrm{~min}^{-1} ; 80{ }^{\circ} \mathrm{C} ; 60 \mathrm{~mol}_{\mathrm{C} 3 \mathrm{H} 6} \mathrm{~mol}_{\mathrm{W}}{ }^{-1}\right.$ $\min ^{-1}, 1$ bar) over $\mathbf{2 a}(\bullet), \mathbf{2 b}(\bigcirc)$ and $\mathbf{3}(\bullet)$. 
